\title{
Characterization of the intronic portion of cadherin superfamily members, common cancer orchestrators
}

\author{
Patrícia Oliveira $^{1,2}$, Remo Sanges ${ }^{3}$, David Huntsman ${ }^{4}$, Elia Stupka ${ }^{5}$ and Carla Oliveira ${ }^{\star, 1,6}$
}

Cadherins are cell-cell adhesion proteins essential for the maintenance of tissue architecture and integrity, and their impairment is often associated with human cancer. Knowledge regarding regulatory mechanisms associated with cadherin misexpression in cancer is scarce. Specific features of the intronic-structure and intronic-based regulatory mechanisms in the cadherin superfamily are unidentified. This study aims at systematically characterizing the intronic portion of cadherin superfamily members and the identification of intronic regions constituting putative targets/triggers of regulation, using a bioinformatic approach and biological data mining. Our study demonstrates that the cadherin superfamily genes harbour specific characteristics in comparison to all non-cadherin genes, both from the genomic and transcriptional standpoints. Cadherin superfamily genes display higher average total intron number and significantly longer introns than other genes and across the entire vertebrate lineage. Moreover, in the human genome, we observed an uncommon high frequency of MIR (mammalian-wide interspersed repeats) and MaLR (mammalian-wide interspersed repeats, a subtype of LTR) regulatory-associated repetitive elements at $5^{\prime}$-located introns, concomitantly with increased de novo intronic transcription. Using this approach, we identified cadherin intronic-specific sites that may constitute novel targets/triggers of cadherin superfamily expression regulation. These findings pinpoint the need to identify mechanisms affecting particularly MIR and MaLR elements located in introns $\mathbf{2}$ and $\mathbf{3}$ of human cadherin genes, possibly important in the expression modulation of this superfamily in homeostasis and cancer. European Journal of Human Genetics (2012) 20, 878-883; doi:10.1038/ejhg.2012.11; published online 8 February 2012

Keywords: cadherin; cancer; intronic-based regulatory elements; MIR; MaLR; transcription

\section{INTRODUCTION}

Cadherins are transmembrane glycoproteins involved in biological functions from tissue morphogenesis to cancer. ${ }^{1}$ All cadherin proteins share one or more copies of a 110-residue extracellular peptide (cadherin repeat-EC), responsible for mediating calcium-dependent homophilic/heterophilic cell-to-cell adhesion. ${ }^{1}$ Many cadherin superfamily members have been proved or suggested to work as tumour suppressor genes and oncogenes in different cancer contexts. Changes affecting cadherin expression are of particular relevance in epithelial cancers, which constitute approximately $80-90 \%$ of all human cancers. $^{2}$ In this context, the non-homeostatic loss of cellular adhesion is frequently the master trigger for invasion and metastization. ${ }^{3}$ Both genetic and epigenetic changes affecting cadherin genes can occur during carcinogenesis with the single purpose of disturbing cellular adhesion and allowing the escape of cancerous cells from primary tumours to more distant locations. ${ }^{2,4}$

E-cadherin (OMIM*192090), a classical tumour suppressor gene, possesses anti-invasive and anti-metastatic properties, ${ }^{5}$ and the clinical turning point in carcinoma progression and metastasis is mediated by its disruption in $90 \%$ of all epithelial cancers. ${ }^{5,6}$ Although classical gene inactivation (mutation, gene loss and promoter hypermethylation) and transcriptional and post-transcriptional mechanisms (transcription repressors, RNA and protein quality control) hamper normal E-cadherin expression and function, ${ }^{5,7}$ these phenomena are insufficient to explain E-cadherin impairment both in the development and overall tumour progression. Regulation by non-coding RNAs (microRNAs), alternative transcripts (antisense transcripts) and alternative translated isoforms (antagonistic isoforms) have recently emerged as a new layer to explain gene and protein expression alterations, ${ }^{8}$ nevertheless their impact on E-cadherin expression and function control in cancer is still poorly understood. A study by Stemmler et $l^{9}$ have shown that intron 2 of CDH1 (ENSG00000039068), the gene encoding E-cadherin, entailed unknown regulatory sequences required to initiate transcriptional activation and to maintain its expression in mouse embryo differentiated epithelia, highlighting the importance of intronic elements in gene expression regulation.

Another interesting phenomena related with the members of the cadherin superfamily is the so-called cadherin switch, often observed in different types of cancer. ${ }^{10,11}$ For example, E and/or P-cadherin expression is often replaced by $\mathrm{N}$-cadherin in malignant breast and prostate cancers, ${ }^{5}$ and desmocollin 2 expression is replaced by de novo expression of desmocollins 1 and 3 in colorectal cancer. ${ }^{10}$ Regardless of the numerous observations in cadherin superfamily members' misexpression in cancer and other diseases, the mechanisms that control these effects are vastly unknown. Growing evidence attribute protein functional impairment to regulation by or at non-protein coding intronic and intergenic sequences. ${ }^{12}$ Therefore, the aim of this study was to systematically characterize the intronic portion of cadherin

\footnotetext{
${ }^{1}$ Instituto de Patologia e Imunologia Molecular da Universidade do Porto, Rua Dr Roberto Frias, s/n, Porto, Portugal; ${ }^{2}$ Instituto de Ciência Biomédicas Abel Salazar, Largo Professor Abel Salazar, 2, Porto, Portugal; ${ }^{3}$ Stazione Zoologica Anton Dohrn, Villa Comunale, Napoli, Italy; ${ }^{4}$ British Columbia Cancer Agency, Vancouver, British Columbia, Canada; ${ }^{5}$ Center for Translational Genomics and Bioinformatics, San Raffaele Scientific Institute, Via Olgettina 60, Milan, Italy; ${ }^{6}$ Faculdade de Medicina da Universidade do Porto, Alameda Professor Hernâni Monteiro, Porto, Portugal

*Correspondence: Dr C Oliveira, Cancer Genetics, Instituto de Patologia e Imunologia Molecular da Universidade do Porto, Rua Dr Roberto Frias, s/n, Porto 4200-465, Portugal. Tel: +351 225570 700; Fax: +351 225570 799; E-mail: carlaol@ipatimup.pt
}

Received 25 August 2011; revised 20 December 2011; accepted 6 January 2012; published online 8 February 2012 
superfamily members, in order to identify regions constituting putative targets of regulation. Using a bioinformatic approach, mining current gene annotation data, ${ }^{13}$ as well as biological data from the ENCODE project, ${ }^{14}$ we investigated genomic and transcriptional intronic-related features of this gene superfamily.

\section{MATERIALS AND METHODS}

Selection of cadherin and non-cadherin genes across species - the seven-domain approach

Using the Ensembl database $(\mathrm{v} 52, \mathrm{v} 60)^{15}$ and InterPro annotation of protein domains, ${ }^{16}$ a keyword search was performed in order to obtain all domains that are present within cadherins from six species that have an extensive genomic infrastructure (such as expressed sequence tag (EST) and cDNA collections) and high coverage after genome sequencing/assembly: Homo sapiens, Mus musculus, Gallus gallus, Danio rerio, Xenopus tropicalis and Ciona intestinalis. These six species provide a general view on chordate evolution starting from $C$. intestinalis (commonly used to explore the evolutionary origins of the chordate lineage ${ }^{17}$ ) up to $H$. sapiens. The following keywords were used based on cadherin superfamily literature information: cadherin, protocadherin, desmosome, desmoglein. Seven domains were thus obtained: IPR000233; IPR002126; IPR006644; IPR009122; IPR013164; IPR014868; and IPR015919. Using PERL scripts and Ensembl API, we collected all non-repeated protein-coding genes, which contained at least one of the seven domains selected as well as both an ATG and STOP codons (data set A). The canonical transcript for each gene was selected based on three criteria in the following order: coding sequence length; number of exons; and transcript length (Supplementary Table 1). For each species, all protein-coding genes that were not selected as cadherins (absent from data set A, given the total absence of the seven InterPro domains selected) were classified as non-cadherin genes, which were used as reference for comparisons with the cadherin superfamily. The same selection criteria were used to select a canonical transcript for each non-cadherin gene. A new cadherin data set (data set B) of genes corresponding to proteins studied by van Roy and group ${ }^{18}$ was built by manual identification (NCBI database) and using PERL scripts (to inquire Ensembl database).

\section{Length class and longest $v s$ non-longest division of introns}

To calculate intron density as a function of intron length, all cadherin and non-cadherin introns extracted from Ensembl were partitioned into three length classes: introns were ranked according to their size and subsequently clustered, such that each length class possessed the same total nucleotide number (bp), which corresponds to one-third of the sum of the length of all introns extracted. ${ }^{19}$ This ranking and clustering was done for three species given the extensive cDNA evidence ( $H$. sapiens, $M$. musculus, D. rerio), and distinct length classes were obtained, given interspecies intronic variation (Supplementary Table 3). After ranking and clustering all introns from each species, each intron was sorted depending on being annotated to a cadherin or non-cadherin gene. All introns were also sorted within each gene depending on being the longest of all introns or one of the non-longest introns. Only genes with at least two introns were used in this part of the sorting analysis. Four groups were thus obtained: cadherin longest introns; cadherin non-longest introns; non-cadherin longest introns; and non-cadherin non-longest introns.

\section{Non-coding sequence conservation analysis}

To determine intronic sequence conservation within cadherin and non-cadherin introns, all available data on mammalian conservation from the Ensembl database $^{15}$ was used. The frequency of genomic evolutionary rate profiling (GERP) constrained elements across 12 mammalian species was analysed to inquire intronic sequence conservation (obtained using Ensembl's EnredoPecan-Ortheus pipeline on 12 eutherian mammals ${ }^{20}$ ). Cadherin and non-cadherin introns from each of the three length classes described were analysed separately. Moreover, both all cadherin and non-cadherin introns and only the longest introns were analysed. A data set was also produced by randomly collecting 1000 sets of 1000 non-cadherin introns, which were also analysed in terms of sequence conservation across mammalians (random data set). For each length class, an average frequency of GERP constrained elements was computed and plotted. For the random data set, the average frequency of GERP constrained elements added or subtracted by 3 SDs was calculated and plotted, thus allowing graphical assessment of significant differences in mammalian conservation.

\section{Total intron number analysis}

With Ensembl API and PERL scripts, the total number of introns in each selected canonical transcript was extracted from the six species selected previously (cadherins and non-cadherins). The resulting distributions were plotted and compared using the Wilcoxon rank-sum test ${ }^{21}$ and the $P$-values corrected using the Bonferroni correction. Three adhesion-related families were selected following the same strategy as for cadherin data set A: $\mathrm{ADAM}^{22}$ (IPR006586-44 genes); integrins ${ }^{23}$ (INTs, IPR000413-19 genes); and tyrosine kinases $^{24}$ (TKs, IPR008266-99 genes). Three families unrelated with cellular adhesion were also selected: $\mathrm{ARF}^{25}$ (IPR006688-106 genes); $\mathrm{MHC}^{26}$ (IPR011162-90 genes); and POU 27 (IPR013847-102 genes). From within all adhesion-related genes $(n=162$ genes, derived from ADAM, INT and TK families), adhesion-unrelated genes ( $n=298$ genes, derived from ARF, MHC and POU families), 1000 randomized sets, of 104 genes each, were selected (because data set A included 104 genes). The total intron number of each gene from each family/data set was extracted using the Ensemb ${ }^{15}$ database. For the randomized data sets, we then calculated the overall average intron number, the minimum, maximum and average inter-quartile range (IQR). ${ }^{28}$

Intron length analysis across cadherin and non-cadherin introns With Ensembl API and PERL scripts we extracted the length of all introns present in each selected canonical transcript from six selected species. Cadherin (data set A) and non-cadherin introns were separated according to the three length classes described and length status (longest and non-longest). The number of introns in each group (length class and length status) was computed. The number of introns in each group was compared (cadherins longest introns vs non-cadherins longest introns; cadherins non-longest introns vs non-cadherins non-longest introns). The Test of Equal or Given Proportions ${ }^{21}$ was performed and the $P$-value corrected using the Bonferroni correction. The length of the longest intron and the average length of all non-longest introns were calculated for each cadherin (data set A) and non-cadherin gene. The distributions obtained for the lengths of longest introns from cadherin and non-cadherin genes were compared. The same was done for the average lengths of non-longest introns. The comparisons were done using the Wilcoxon ranksum test, ${ }^{21}$ and were performed for all cadherin and non-cadherin introns from the six species queried. The average ratio between longest introns from cadherins and non-cadherins was calculated: (1) all average lengths for longest introns obtained previously were summed and divided by the total number of cadherin longest introns annotated; (2) the same was done for non-cadherin introns; (3) these two values were divided thus obtaining the overall ratio between cadherin and non-cadherin longest introns. The same was done for non-longest introns.

\section{Analysis of the position of the longest intron}

Using PERL scripts and the Ensembl database (v52, v60), the position of the longest intron was assessed in both cadherin (data set A) and non-cadherin genes: ie, position 1 corresponds to the first intron in the gene (most $5^{\prime}$ ) immediately after exon 1 . The Wilcoxon rank-sum test ${ }^{21}$ was selected to compare the obtained intron position distributions. The same was done only for length class 3 longest introns.

\section{Analysis of the frequency of regulatory elements and repetitive elements}

Several regulatory and repetitive elements were analysed in terms of frequency. Data for both these types of elements were obtained using the Ensembl database (v52, elements inquired in Supplementary Tables 8 and 9). The analysis was done by comparing the frequency of base pairs overlapping each feature studied in human cadherin and non-cadherin introns as well as with the random data set of introns described previously. Introns were studied 
separately according to the three length classes described earlier. Moreover, introns were studied as a whole as well as separated into longest and non-longest.

\section{Intron length, repetitive elements and CAGE data analysis}

Introns from each of assessed position were analysed in terms of length and number of $A l u, M I R$ and MaLR elements normalized to intron length of origin. Introns were also assessed in terms of intronic transcription, using the available data from the CAGE experiments within the ENCODE project ${ }^{14,29,30}$ (files analysed are described in Supplementary Table 10). We focused on CAGE data collected using the 'normal' lymphoblastoid cell line GM12878: long poly-Anegative RNA from both genomic strands and from the nucleic and cytosolic fractions. For each intron position, cadherin and non-cadherin CAGE tag distributions were computed, all of which normalized to intron length of origin, thus avoiding intron length-related bias. The obtained distributions were compared using the Wilcoxon rank-sum test ${ }^{21}$ and $P$-values corrected using the Bonferroni correction.

\section{RESULTS}

\section{Selection of cadherin superfamily members}

To characterize the genetic architecture of as many members as possible belonging to the cadherin superfamily, we generated criteria that could successfully gather all genes within this superfamily, using the Ensembl database. ${ }^{13,15}$ We combined literature data and protein domain databases (InterPro ${ }^{16}$ ) to collect genes that coded for protein sequences currently classified as cadherins from six distinct genomes, resulting in seven distinct cadherin-related InterPro domains. Selected genes were further curated to assure that only protein-coding genes were selected and included in data set A (Supplementary Table 1). All protein-coding genes in each genome that were not selected for the cadherins' data set A were grouped in a control data set named noncadherins. To validate data set A, we compared it with a previously published data set (data set B) based on cadherin superfamily proteinrelated information. ${ }^{18}$ For the $H$. sapiens genome, $90 \%$ of cadherins in data set $\mathrm{B}$ matched those of data set $\mathrm{A}$. The remaining $10 \%$, corresponded to (1) four protein sequences whose genomic locus we were not able to identify and; (2) four protein sequences that either our approach did not consider to be a cadherin (ie, it did not possess any of the seven InterPro domains selected) or were excluded from data set A given the absence of an annotated ATG/STOP codon. Five of the six genomes analysed, with the exception of $X$. tropicalis, produced a high overlap between data sets A and B (Supplementary Table 2).

\section{Cadherin introns do not exhibit significant intronic sequence conservation}

We investigated whether the DNA sequence of cadherin introns was being conserved across vertebrate species. We compared the intronic sequence conservation (Genomic Evolutionary Rate Profiling constrained elements) across 12 mammalian species ${ }^{15,31}$ by studying 3 separated groups of human introns: (1) cadherin introns (data set A); (2) non-cadherin introns; and (3) 1000 data sets of 1000 randomly chosen non-cadherin introns (random data set). Sequence conservation was analysed by separating all cadherin and non-cadherin introns into three length classes, each encompassing the same number of nucleotides. ${ }^{19}$ Length classes were named $1-3$, with the latter encompassing the longer introns in each genome (Supplementary Table 3 ). By analysing separately each intron length class, we aimed at preventing any length-related bias. In addition, given that most genes in several genomes present their longest intron at the most $5^{\prime}$-end of the gene and that $5^{\prime}$-introns frequently encompass important non-coding regulatory elements, ${ }^{32}$ we also analysed the sequence conservation of the longest intron from each cadherin and non-cadherin genes. We observed that cadherin introns' frequency of constrained elements was never significantly distinct from that of non-cadherin introns. This was valid for all length classes and for longest introns. In addition, no significant differences were observed in terms of intronic sequence conservation when comparing cadherin introns with the collected random data sets (Supplementary Figure 1).

\section{Cadherins show a higher average intron number than non-} cadherins as well as a particular pattern of intron distribution Using the total intron number as a measure of gene architecture, we have observed that overall, the cadherin superfamily of genes presented a higher average intron number than non-cadherin genes in all species analysed ( $>11$ introns for cadherins and $<9$ for noncadherins, $P$-value ranging from $2.76 \mathrm{E}-08$ to $1.15 \mathrm{E}-02$, Figure 1 , Supplementary Figure 2 and Supplementary Table 4). Moreover, the distribution of total intron number was wider in comparison to noncadherin genes and particularly prominent in human and mouse genomes (Figure 1 and Supplementary Figure 2). The analysis of the

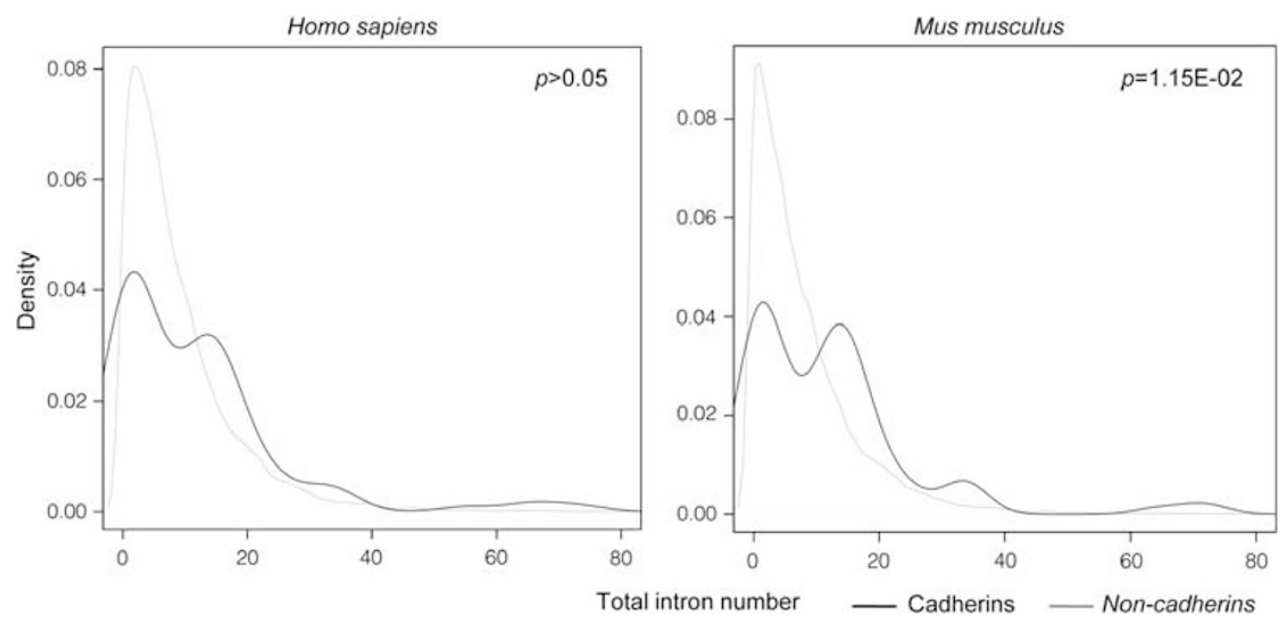

Figure 1 Total intron number distribution in human and mouse non-cadherin genes and cadherin superfamily genes. The grey full line represents non-cadherin genes and the black full line represents cadherin genes (data set $A$ ). Values in the top right corner correspond to $P$-values obtained when comparing data set A against non-cadherins. 
distribution of total intron number revealed that while non-cadherins displayed a single and highly enriched peak corresponding to genes with less than 10 introns, the intron number distribution of cadherin genes resulted in four distinct peaks (human and mouse genomes): peak 1 corresponded to <10 introns; peak 2 corresponded to 12-20 introns; peak 3 corresponded to 30-40 introns; and peak 4 corresponded to 50-80 introns (Figure 1). Concerning cadherin genes found in non-mammalian species (except $C$. intestinalis), the peaks observed were less distinct than in mammals, yet still present and the total intron number in cadherins was also significantly distinct from non-cadherin genes ( $P$-value ranging from $1.46 \mathrm{E}-03$ to $2.76 \mathrm{E}-08$, Supplementary Figure 1 and Supplementary Table 4).

To understand whether the wider range of total intron number resulted mainly from the comparison of a single family of genes against all genes in the genome, we compared the total intron number in cadherins to that of other human gene families/sets: (1) adhesionrelated $\mathrm{ADAM},{ }^{22} \mathrm{INT}^{23,24}$ and $\mathrm{TKs} ;{ }^{24}$ (2) adhesion-unrelated $\mathrm{ARF},{ }^{25}$ $\mathrm{MHC}^{26}$ and $\mathrm{POU}^{27}$ ); (3) 1000 randomized sets of 104 human genes selected from within all adhesion-related genes and from within all adhesion-unrelated genes. By comparing intron number distribution with the IQRs (which measures data dispersion ${ }^{28}$ ) from cadherin genes with those of the remaining families of genes, we observed that while cadherins displayed an $\mathrm{IQR}=15.3$, adhesion-related families displayed an $\mathrm{IQR}<8.5$, adhesion-unrelated families of genes displayed an IQR $\leq 3$ and random data sets exhibited average IQRs not higher than 9.9 (Supplementary Table 5). These results showed that cadherin genes displayed a wider total intron number distribution in comparison with all other families/data sets, supporting our previous observation for comparison with non-cadherin genes, and proved that cadherins are in fact a particular family of genes in terms of total intron number distribution.

\section{Cadherin genes have significantly longer introns than other genes in the vertebrate lineage}

The assessment of cadherin genetic structure revealed that cadherin family members such as $C D H 1, C D H 2$ and $C D H 3$ presented unusually large introns. ${ }^{9,33}$ Given the growing evidence of functional regulatory features located within introns, ${ }^{34,35}$ we analysed whether the cadherin superfamily of genes displayed significantly longer introns than other genes in the genomes of $H$. sapiens, $M$. musculus and D. rerio, using the three length classes previously described (Supplementary Table 3). We further analysed separately the longest intron from each gene and all other introns, classified as non-longest. This comparison showed a significant enrichment of all cadherin introns (both longest and non-longest) in length class 3 for all three genomes ( $P$ ranging from $1.32 \mathrm{E}-15$ to $6.57 \mathrm{E}-07$, Figure 2, Supplementary Figure 3 and Supplementary Table 6). All non-cadherin introns analysed (except $D$. rerio non-cadherin longest introns) in turn were significantly more present in length class 1 ( $P$ ranging from $1.32 \mathrm{E}-15$ to $2.08 \mathrm{E}-06$, Supplementary Table 6). These data indicate that cadherin introns are significantly longer than those of non-cadherin genes (regardless of the longest/non-longest status, data not shown) and that this feature is conserved, being therefore potentially relevant to the entire vertebrate lineage. We further calculated the average length of longest introns from cadherin and non-cadherin genes and computed a ratio (average length of longest cadherin introns divided by average length of longest non-cadherin introns). The same was done for non-longest introns. The distributions obtained were compared, and it revealed that five of six species displayed significantly higher ratios either for cadherin longest and non-longest introns ( $P$ ranging from $1.96 \mathrm{E}-11$ to $4.92 \mathrm{E}-02$, Supplementary Table 7). An average length ratio per species was

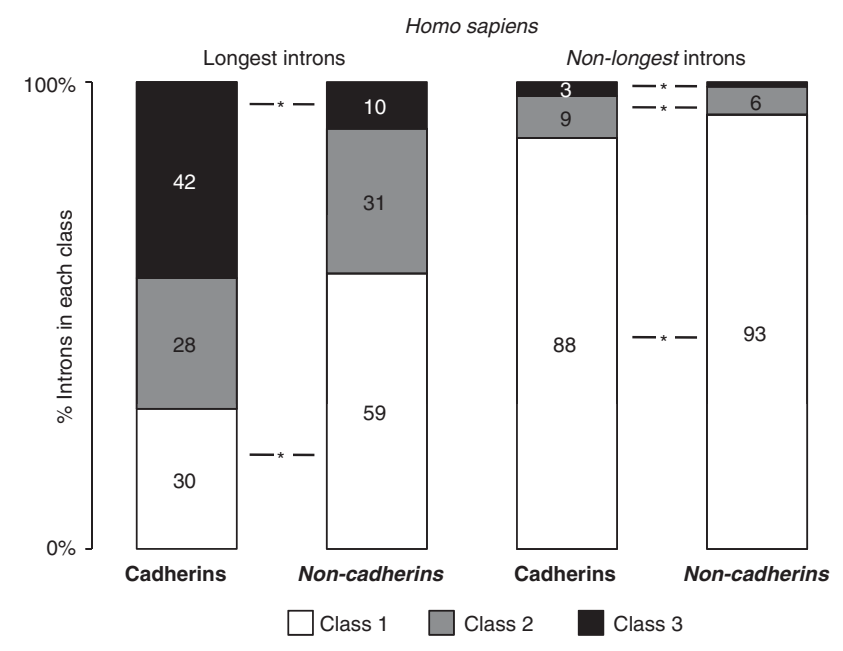

Figure 2 Distribution across length classes of human cadherin and non-cadherin longest and non-longest introns. White columns correspond to length class 1 introns; grey columns correspond to length class 2 introns; and black columns correspond to length class 3 introns. Data labels refer to the percentage of introns in each length class, type of gene and length status. Asterisks stand for significantly distinct comparisons $(P<0.05)$.

also computed and plotted to ease visualization (Supplementary Figure 4). The higher length ratios for cadherin introns were observed across all vertebrate species analysed, but not in C. intestinalis, an invertebrate species, reinforcing that cadherin's intron length may be a pervasive vertebrate feature.

Cadherin longest introns are positioned preferably at the $5^{\prime}$ start of the gene and are MIR and MaLR rich and Alu poor

Prior reported studies indicated that in eukaryotes the first introns (positioned closer to the $5^{\prime}$-end of genes) tend to be longer, ${ }^{32}$ and often harbour relevant regulatory elements essential for gene expression control. ${ }^{19,32,34}$ Therefore, we next investigated the position of the longest intron of cadherins in comparison to the annotated transcription start site (TSS). Our data indicated that the majority of cadherins' longest introns (regardless of class) were positioned in the closest $5^{\prime}$-position in relation to the TSS, as were non-cadherin longest introns in all species analysed $(P>0.05$, Supplementary Figure 5 and data not shown). These observations pointed out that the position of the longest introns in cadherins followed the known trend in several vertebrate genomes inquired.

We next tested whether 5'-located long introns would accommodate important regulatory-associated annotated features, and determined the frequency of such features within cadherin introns. We analysed the presence of (1) DNAsel hypersensitive sites, which mark for accessible chromatin; ${ }^{36}$ (2) several histone methylation and acetylation marks, which commonly underlie promoter elements; ${ }^{37}$ (3) ESTs, which mark for transcription; ${ }^{38}$ and (4) DNA repetitive sequences, which have been shown to impact gene regulation. ${ }^{39,40} \mathrm{We}$ observed that, for all above-mentioned regulatory features, human cadherin introns displayed similar frequencies in comparison to all non-cadherin introns and to the random data set $(P>0.05$, data not shown), with the exception of DNA repetitive sequences. Human cadherin and non-cadherin introns revealed significant differences in terms of frequency of two specific families of repeats: short interspersed nuclear elements (SINE) and long terminal repeats (LTRs). SINE elements, in particular Alu and MIR (mammalian-wide interspersed repeats), were found to be present in a significantly distinct 
manner in cadherin introns: (1) Alu elements were significantly less frequent in cadherin introns than in non-cadherin introns $(P=3.94 \mathrm{E}-14$ and $1.14 \mathrm{E}-13$ for length classes 1 and 2, respectively, Supplementary Figure 6A); (2) MIR elements were significantly more frequent in cadherin introns $(P=1.38 \mathrm{E}-04$ for length class 1 , Supplementary Figure 6B) in comparison with non-cadherin introns. For LTR elements, a significantly higher frequency of MaLR (mammalianwide interspersed repeats, a subtype of LTR) was observed in both cadherins' smaller and longer introns in comparison with non-cadherin introns $(P=1.05 \mathrm{E}-03$ and 3.57E-02 for length classes 1 and 3, respectively, Supplementary Figure 6C).

In order to integrate intron length and repeat frequency, we reanalysed all these features in terms of intron positioning. We observed that in terms of intron length, cadherin longest introns were significantly longer than non-cadherin longest introns found at positions $1,2,3,5$ and 7 ( $P$ ranging from $9.93 \mathrm{E}-09$ to $3.92 \mathrm{E}-02$, Figure 3). Next, we observed that both MIR and MaLR elements' frequency (normalized to intron length) was found to be significantly enriched in introns positioned closer to the $5^{\prime}$ start of cadherins in comparison with non-cadherin genes. In particular, cadherin introns in positions 2, 3, 7 and 8 were significantly enriched for MIR elements ( $P$ ranging from $2.30 \mathrm{E}-06$ to $4.21 \mathrm{E}-02$, Figure 3 ) and cadherin introns in positions $2,3,6,7,8$ and 9 were significantly enriched in MaLR elements ( $P$ ranging from $1.00 \mathrm{E}-09$ to $7.58 \mathrm{E}-03$, Figure 3 ). Cadherin introns were significantly impoverished in Alu elements in position 10 in comparison with non-cadherin introns $(P=3.99 \mathrm{E}-03$, Figure 3).

\section{Human cadherin introns exhibit significantly increased MIR and MaLR frequency with simultaneous increased intronic transcription}

MIR and MaLR repeat elements are involved in genome novelty by alternative regulation phenomena as well as by promoting exonization. ${ }^{39-41}$ Therefore, we next analysed whether the unusual frequency of Alu, MIR and MaLR elements within cadherin introns was

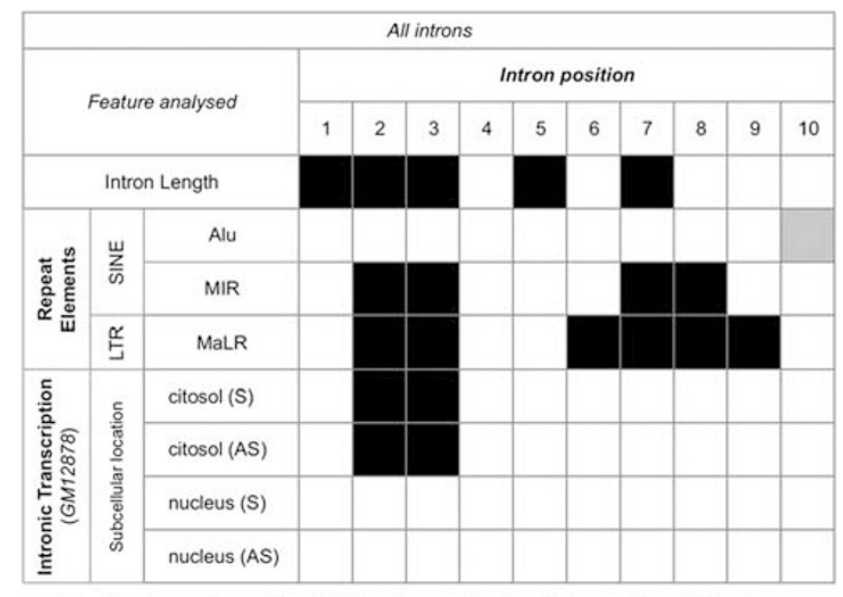

Cadherin enrichment $(p<0.05) \square$ Non-cadherin enrichment $(p<0.05) \square p>0.05$

Figure 3 Comparison of intron length, normalized repeat frequency and normalized intronic transcription between human cadherin and non-cadherin introns. Human cadherin and non-cadherin introns were separated according to their position in each corresponding gene (position 1 corresponds to the most $5^{\prime}$ intron). Black squares correspond to a significantly enriched feature for cadherin introns $(P<0.05)$; grey squares correspond to a significantly enriched feature for non-cadherin introns $(P<0.05)$; and white squares correspond a non-significantly distinct feature $(P>0.05)$. correlated with differential intronic transcription arising from cadherin introns.

To assess cadherin intronic transcription, we mined the data from the pilot stage of the ENCODE project, ${ }^{14}$ in particular data obtained by $5^{\prime}$ cap analysis gene expression (CAGE) performed by Carninci and group $^{29}$ at the RIKEN Institute. This technique allowed for the detection of new TSS, and we focused on the data obtained using RNA extracted from cytosolic and nucleic fractions of the lymphoblastic human normal cell line GM12878 to assess transcription. ${ }^{14}$ We verified that cadherin introns at positions 2 and 3 displayed a significant TSS enrichment in the cytosolic fraction of the cell line GM12878, arising from both RNA strands ( $P$ ranging from $1.51 \mathrm{E}-04$ to $4.01 \mathrm{E}-02$, Figure 3 ). Taken together, a correlation was observed for cadherin introns found at positions 2 and 3, for which cadherin introns were significantly longer, carried increased frequency of MIR and MaLR elements and increased levels of novel transcription initiation than non-cadherin introns (Figure 3).

\section{DISCUSSION}

The herein presented systematic characterization of the cadherin superfamily of genes encompassed the analysis of the intron sequence conservation; total intron number as a measure of gene structure; intron length and positioning; annotated regulatory and putative regulatory elements; and de novo intronic transcription initiation. We observed that this superfamily displays a higher average intron number than non-cadherins, a particular pattern of intron distribution, and significantly longer introns than the rest of the genome, throughout the vertebrate lineage, emphasizing an overall gene structure conservation in cadherins genes without intronic sequence conservation. This type of analysis has not been reported for other gene families and therefore it is difficult to extrapolate its implications. Nevertheless, the maintenance of many and large fragments of intronic DNA across evolution may indicate an unanticipated importance of these specific structural features. In fact, lack of sequence conservation does not imply lack of functionality of underlying elements and may rather derive from a rapid sequence evolution crucial for species adaptation. ${ }^{12}$ We also observed that the unusually long introns were preferably located in the $5^{\prime}$ start of cadherin genes in line with what is currently described for overall genomes. ${ }^{32}$ In fact, first introns are thought to encompass relevant (if not fundamental) regulatory elements. ${ }^{19,32,34}$ This could therefore support the fact that cadherin introns' length and total intron number have been conserved during evolution to maintain essential underlying regulatory features.

The search for regulatory elements was only performed on the human genome due to the larger bioinformatic data availability (derived from projects such as ENCODE ${ }^{14}$ ) and revealed that neither the cadherins' intron sequence was being significantly conserved nor typical insulator/enhancer underlying elements, histone methylation marks or even accessible chromatin areas. Nevertheless, cadherin introns carried significantly more MIR and MaLR repetitive sequences and less Alu elements than all other genes in the human genome. All these repetitive elements are known to be involved in novel regulatory mechanisms and in exonization. ${ }^{39,40}$ Given that the occurrence of exonization leads to the creation of new transcriptionally active regions, we analysed cadherin introns in terms of intron position and observed introns in positions 2 and 3 were not only longer but harboured a concomitant increased frequency of MIR, MaLR and CAGE tags in comparison with the rest of the human genome. This clearly suggests that the long cadherin introns at these two positions may in fact encode novel transcribed regulatory elements associated with MIR and MaLR repetitive elements. Supporting this observation, 
it has been shown that $\mathrm{CDH} 1$ intron 2 entailed unknown regulatory sequences required to initiate transcriptional activation and to maintain its expression in mouse embryo differentiated epithelia. ${ }^{9}$ In fact, several other studies have also revealed the relevance of the presence of introns (and consequently of putative intron-based elements) for basic biological processes such as mRNA stability and gene expression. In Arabidopsis thaliana, the removal of introns from the ERECTA gene leads to a dramatic decrease in its mRNA production, which is in addition much less stable and prone to degradation. ${ }^{42}$ In the green alga Ostreococcus lucimarinus, a positive correlation between intron presence and increased gene expression has been observed. ${ }^{43}$ Moreover, following the same idea, the few annotated intron-containing genes in yeast are responsible for almost one-third of all mRNA transcription, ${ }^{44}$ and both human and plant intron-containing genes encode for more stable mRNA transcripts than genes without introns. ${ }^{45}$

In conclusion, our study demonstrates that the cadherin superfamily of genes harbours highly specific characteristics from the genomic and transcriptional standpoints, namely high frequency of specific repetitive elements within cadherin $5^{\prime}$-located long introns combined with an unusual frequency of novel transcription initiation. These findings lay the ground for discovering novel areas important in fine-tuning the expression of this gene family as well as intronic-based regulatory mechanisms, particularly in introns 2 and 3, important for expression of the cadherin superfamily of genes in biological events such as cadherin switching or cadherin gene loss/functional impairment in homeostasis and disease.

\section{CONFLICT OF INTEREST}

The authors declare no conflict of interest.

\section{ACKNOWLEDGEMENTS}

We acknowledge the Portuguese Foundation for Science and Technology (FCT) for: project funding (PTDC/SAU-GMG/72168/2006, PTDC/SAU-GMG/ 110785/2009), PhD grant to PO (SFRH/BD/32984/2006) and salary support to Carla Oliveira from POPH - QREN/Type 4.2, European Social Fund and Portuguese Ministry of Science and Technology (MCTES). PO is a PhD student of the Graduate Program in Areas of Basic and Applied Biology (GABBA, 10th edition).

1 Halbleib JM, Nelson WJ: Cadherins in development: cell adhesion, sorting, and tissue morphogenesis. Genes Dev 2006; 20: 3199-3214.

2 Christofori G, Semb H: The role of the cell-adhesion molecule E-cadherin as a tumour suppressor gene. Trends Biochem Sci 1999; 24: 73-76.

3 Hanahan D, Weinberg RA: Hallmarks of cancer: the next generation. Cell 2011; 144 646-674.

4 Nieto MA: Epithelial-mesenchymal transitions in development and disease: old views and new perspectives. Int J Dev Biol 2009; 53: 1541-1547.

5 Berx G, van Roy $F$ : Involvement of members of the cadherin superfamily in cancer. Cold Spring Harb Perspect Biol 2009; 1: a003129.

6 Vleminckx K, Vakaet Jr L, Mareel M, Fiers W, van Roy F: Genetic manipulation of E-cadherin expression by epithelial tumor cells reveals an invasion suppressor role. Cell 1991: 66: 107-119.

7 Cano A, Perez-Moreno MA, Rodrigo I et al: The transcription factor snail controls epithelial-mesenchymal transitions by repressing E-cadherin expression. Nat Cell Biol 2000; 2: 76-83.

8 Taft RJ, Pang KC, Mercer TR, Dinger M, Mattick JS: Non-coding RNAs: regulators of disease. J Pathol 2010; 220: 126-139.

9 Stemmler MP, Hecht A, Kemler R: E-cadherin intron 2 contains cis-regulatory elements essential for gene expression. Development 2005; 132: 965-976.

10 Khan K, Hardy R, Haq A, Ogunbiyi O, Morton D, Chidgey M: Desmocollin switching in colorectal cancer. Br J Cancer 2006; 95: 1367-1370.
11 Tomita K, van Bokhoven A, van Leenders GJ et al: Cadherin switching in human prostate cancer progression. Cancer Res 2000; 60: 3650-3654.

12 Pang KC, Frith MC, Mattick JS: Rapid evolution of noncoding RNAs: lack of conservation does not mean lack of function. Trends Genet 2006; 22: 1-5.

13 Flicek P, Aken BL, Ballester B et al: Ensembl's 10th year. Nucleic Acids Res 2010; 38: D557-D562.

14 Consortium EP: Identification and analysis of functional elements in $1 \%$ of the human genome by the ENCODE pilot project. Nature 2007; 447: 799-816.

15 Flicek P, Aken BL, Beal K et al: Ensembl 2008. Nucleic Acids Res 2008; 36: D707-D714.

16 Hunter S, Apweiler R, Attwood TK et al: InterPro: the integrative protein signature database. Nucleic Acids Res 2009; 37: D211-D215.

17 Dehal P, Satou Y, Campbell RK et al: The draft genome of Ciona intestinalis: insights into chordate and vertebrate origins. Science 2002; 298: 2157-2167.

18 Hulpiau P, van Roy F: Molecular evolution of the cadherin superfamily. Int J Biochem Cell Biol 2009; 41: 349-369.

19 Sironi M, Menozzi G, Comi GP, Cagliani R, Bresolin N, Pozzoli U: Analysis of intronic conserved elements indicates that functional complexity might represent a major source of negative selection on non-coding sequences. Hum Mol Genet 2005; 14: 2533-2546.

20 Hubbard TJ, Aken BL, Ayling S et al: Ensembl 2009. Nucleic Acids Res 2009; 37: D690-D697.

21 R_Development_Core_Team: R: A Language and Environment for Statistical Computing. R Foundation for Statistical Computing 2008.

22 Edwards DR, Handsley MM, Pennington CJ: The ADAM metalloproteinases. Mol Aspects Med 2008; 29: 258-289.

23 Zaidel-Bar R, Geiger B: The switchable integrin adhesome. J Cell Sci 2010; 123: $1385-1388$.

24 Zhao J, Guan JL: Signal transduction by focal adhesion kinase in cancer. Cancer Metastasis Rev 2009; 28: 35-49.

25 Boman AL, Kahn RA: Arf proteins: the membrane traffic police? Trends Biochem Sci 1995; 20: 147-150.

26 Clark DA, Chaouat G, Wong K, Gorczynski RM, Kinsky R: Tolerance mechanisms in pregnancy: a reappraisal of the role of class I paternal MHC antigens. Am J Reprod Immunol 2010; 63: 93-103.

27 Andersen B, Rosenfeld MG: POU domain factors in the neuroendocrine system: lessons from developmental biology provide insights into human disease. Endocr Rev 2001; 22: $2-35$.

28 Upton G, Cook I: Understanding Statistics. Great Britain: Oxford University Press, 1996.

29 Kodzius R, Kojima M, Nishiyori $\mathrm{H}$ et al: CAGE: cap analysis of gene expression. Nat Methods 2006; 3: 211-222.

30 Rhead B, Karolchik D, Kuhn RM et al: The UCSC Genome Browser database: update 2010. Nucleic Acids Res 2010; 38: D613-D619.

31 Cooper GM, Stone EA, Asimenos G, Green ED, Batzoglou S, Sidow A: Distribution and intensity of constraint in mammalian genomic sequence. Genome Res 2005; 15 : 901-913

32 Bradnam KR, Korf I: Longer first introns are a general property of eukaryotic gene structure. PLoS One 2008; 3: e3093.

33 van Roy F, Berx G: The cell-cell adhesion molecule E-cadherin. Cell Mol Life Sci 2008; 65: 3756-3788.

34 Mattick JS: The genetic signatures of noncoding RNAs. PLoS Genet 2009; 5: e1000459.

35 Nagano T, Mitchell JA, Sanz LA et al: The Air noncoding RNA epigenetically silences transcription by targeting G9a to chromatin. Science 2008; 322: 1717-1720.

36 Sabo PJ, Hawrylycz M, Wallace JC et al: Discovery of functional noncoding elements by digital analysis of chromatin structure. Proc Natl Acad Sci USA 2004; 101: 16837-16842.

37 Reik W: Stability and flexibility of epigenetic gene regulation in mammalian development. Nature 2007; 447: 425-432.

38 Adams MD, Kelley JM, Gocayne JD et al: Complementary DNA sequencing: expressed sequence tags and human genome project. Science 1991; 252: 1651-1656.

39 Krull M, Petrusma M, Makalowski W, Brosius J, Schmitz J: Functional persistence of exonized mammalian-wide interspersed repeat elements (MIRs). Genome Res 2007; 17: 1139-1145.

40 Piriyapongsa J, Polavarapu N, Borodovsky M, McDonald J: Exonization of the LTR transposable elements in human genome. BMC Genomics 2007; 8: 291.

41 Prudhomme S, Oriol G, Mallet F: A retroviral promoter and a cellular enhancer define a bipartite element which controls env ERVWE1 placental expression. J Virol 2004; 78: 12157-12168.

42 Karve R, Liu W, Willet SG, Torii KU, Shpak ED: The presence of multiple introns is essential for ERECTA expression in Arabidopsis. RNA 2011; 17: 1907-1921.

43 Lanier W, Moustafa A, Bhattacharya D, Comeron JM: EST analysis of Ostreococcus lucimarinus, the most compact eukaryotic genome, shows an excess of introns in highly expressed genes. PLoS One 2008; 3: e2171.

44 Ares Jr M, Grate L, Pauling MH: A handful of intron-containing genes produces the lion's share of yeast mRNA. RNA 1999; 5: 1138-1139.

45 Wang HF, Feng L, Niu DK: Relationship between mRNA stability and intron presence. Biochem Biophys Res Commun 2007; 354: 203-208.

Supplementary Information accompanies the paper on European Journal of Human Genetics website (http://www.nature.com/ejhg) 